\title{
POLYNOMIALS IN ASYMPTOTICALLY FREE RANDOM MATRICES
}

\author{
ROLAND SPEICHER
}

\begin{abstract}
Recent work of Belinschi, Mai and Speicher resulted in a general algorithm to calculate the distribution of any selfadjoint polynomial in free variables. Since many classes of independent random matrices become asymptotically free if the size of the matrices goes to infinity, this algorithm allows then also the calculation of the asymptotic eigenvalue distribution of polynomials in such independent random matrices. We will recall the main ideas of this approach and then also present its extension to the case of the Brown measure of non-selfadjoint polynomials.
\end{abstract}

\section{INTRODUCTION}

Recent work of Belinschi, Mai and Speicher 2 resulted in a general algorithm to calculate the distribution of any selfadjoint polynomial in free variables. Since many classes of independent random matrices become asymptotically free if the size of the matrices goes to infinity, this algorithm applies then also to the calculation of the asymptotic eigenvalue distribution of polynomials in such independent random matrices. Here we will, after first recalling the main ideas of this approach, also address the non-selfadjoint situation and present the results of work in progress of Belinschi, Sniady and Speicher [4. There it is shown that the combination of the ideas from the selfadjoint case with the hermitization method allows to extend our algorithm also to the calculation of the Brown measure of arbitrary polynomials in free variables. It is expected that this Brown measure is then also the limiting eigenvalue distribution of corresponding random matrix problems; however, since the convergence of $*$-moments does not necessarily imply the convergence of Brown measures in the non-normal case, this has to remain as a conjecture for the moment.

\section{The Case of One Matrix}

We are interested in the limiting behaviour of $N \times N$ random matrices for $N \rightarrow$ $\infty$. Let us first recall the well-known situation of one matrix.

Typical phenomena for basic random matrix ensembles are that we have almost sure convergence of the eigenvalue distribution to a deterministic limit and, furthermore, this limit distribution can be effectively calculated.

Talk given at the conference "Random Matrix Theory: Foundations and Applications" in Krakow, Poland 2014.

This work has been supported by the ERC Advanced Grant "Noncommutative Distributions in Free Probability" NCDFP 339760. 
The common analytic tool for calculating this limit distribution is the Cauchy transform. For any probability measure $\mu$ on $\mathbb{R}$ we define its Cauchy transform

$$
G(z):=\int_{\mathbb{R}} \frac{1}{z-t} d \mu(t) .
$$

This is an analytic function $G: \mathbb{C}^{+} \rightarrow \mathbb{C}^{-}$and we can recover $\mu$ from $G$ by Stieltjes inversion formula

$$
d \mu(t)=-\frac{1}{\pi} \lim _{\varepsilon \rightarrow 0} \Im G(t+i \varepsilon) d t .
$$

Quite often, one prefers to work with $-G$, which is called the Stieltjes transform.

For the basic matrix ensembles, the Gaussian and the Wishart random matrices, one can derive quadratic equations for the respective Cauchy transforms and thus get an explicit formula for $G(z)$. In Figure 1 we show the comparision between histograms of eigenvalues and the theoretical limit result given by the Cauchy transform.
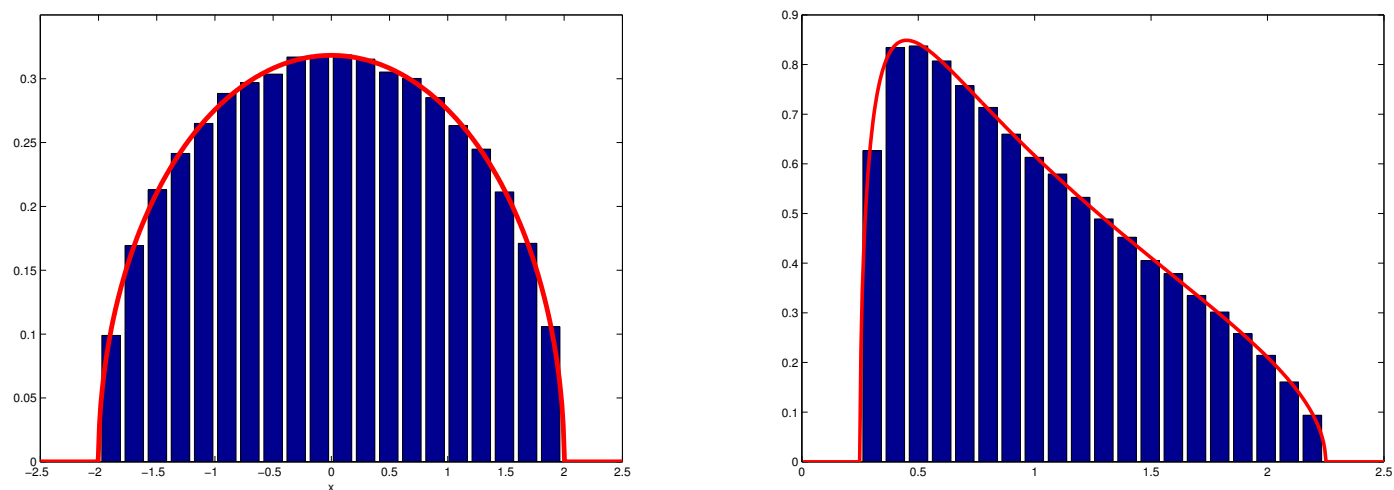

FigURE 1. left: histogram of the eigenvalues of a $4000 \times 4000$ Wigner matrix, compared to Wigner's semicircle, determined by its Cauchy transform $G(z)=\frac{z-\sqrt{z^{2}-4}}{2}$; right: histogram of the eigenvalues of a Wishart matrix $X X^{*}$ for a $3000 \times 12000$ matrix $\mathrm{X}$, compared to the Marchenko-Pastur distribution for $\lambda=1 / 4$, determined by its Cauchy transform $G(z)=$ $\frac{z+1-\lambda-\sqrt{(z-(1+\lambda))^{2}-4 \lambda}}{2 z}$

\section{The Case of Several Independent Matrices}

Instead of one random matrix we are now interested in the case of several independent random matrices. Since there is no meaningful notion of joint eigenvalue distribution of several non-commuting matrices, we are looking instead on the eigenvalue distribution of polynomials in those matrices. For the moment, we restrict to selfadjoint polynomials in selfadjoint matrices. Later we will look on the non-selfadjoint case. So we are interested in the limiting eigenvalue distribution of general selfadjoint polynomials $p\left(X_{1}, \ldots, X_{k}\right)$ of several independent selfadjoint $N \times N$ random matrices $X_{1}, \ldots, X_{k}$. 
Again one observes the typical phenomena of almost sure convergence to a deterministic limit eigenvalue distribution. However, this limit distribution can be effectively calculated only in simple situations.

In Figure 2 we show two such typical situations. The first is the polynomial $p(X, Y)=X+Y$, for independent Wigner matrix X and Wishart matrix Y; in this case, one can derive quite easily the implicit equation $G(z)=G_{\text {Wishart }}(z-G(z))$ for the Cauchy transform $G(z)$ of the limiting distribution of $p(X, Y)$; this subordinated form of equation is quite amenable to numerical solutions via iterations and serves as the model what we can hope for in more general situations. The second case is the polynomial $p(X, Y)=X Y+Y X+X^{2}$, for which no theoretical result has existed up to now.
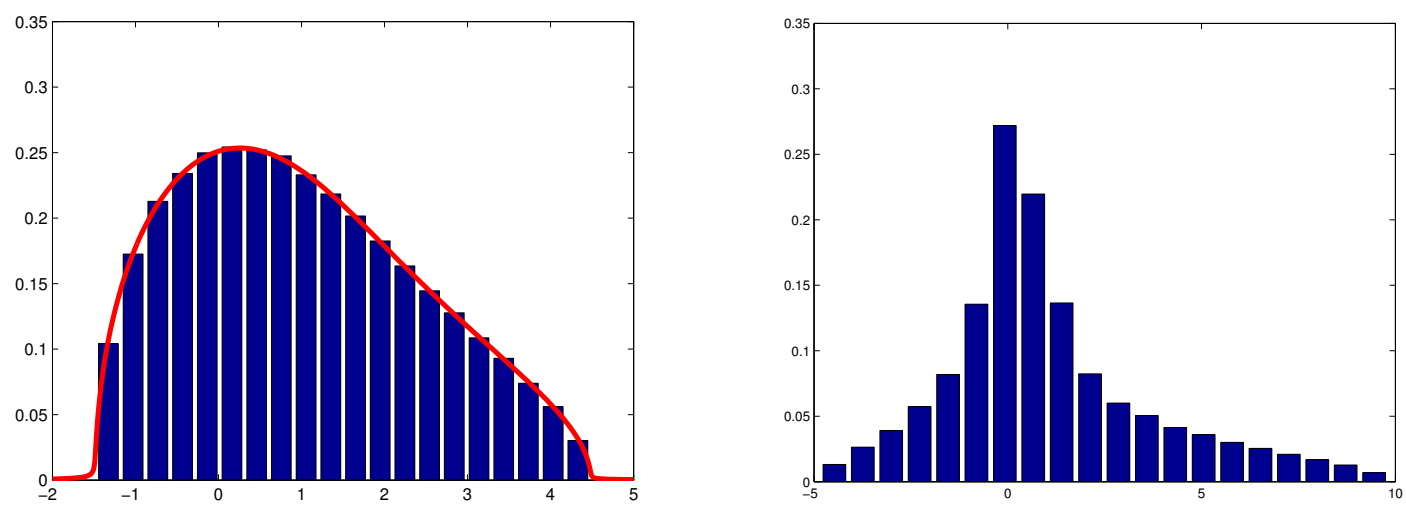

FiguRE 2. Histogram for a generic realization of a $3000 \times 3000$ random matrix $p(X, Y)$, where $X$ and $Y$ are independent Gaussian and, respectively, Wishart random matrices: $p(X, Y)=X+Y$ (left); $p(X, Y)=X Y+Y X+X^{2}$ (right). In the left case, the asymptotic eigenvalue distribution is relatively easy to calculate; in the right case, no such solution was known, this case will be reconsidered in Figure 3

There existed a huge literature on special cases of such polynomials in independent matrices (see, for example, [5, 9, 12, 13, 16, 17, 18]), but up to [2] there has been no algorithm for addressing the general problem.

\section{Asymptotic Freeness of Random Matrices}

The problem of the asymptotic eigenvalue distribution of random matices became linked to free probability theory by the basic result of Voiculescu 20] that large classes of independent random matrices (like Wigner or Wishart matrices) become asymptoticially freely independent.

The conclusion of this is: calculating the asymptotic eigenvalue distribution of polynomials in such matrices is the same as calculating the distribution of polynomials in free variables. The latter is an intrinsic problem within free probability theory. 
We want to understand the distribution of polynomials in free variables. What we understand quite well, by the analytic theory of free convolution, is the sum of free selfadjoint variables. So we should reduce the problem of arbitrary polynomials to the problem of sums of selfadjoint variables.

This sounds like a quite ambitious goal, but it can indeed be achieved. However, there is a price to pay: we have to go over to an operator-valued frame.

\section{The Operator-Valued Setting}

Let $\mathcal{A}$ be a unital algebra and $\mathcal{B} \subset \mathcal{A}$ be a subalgebra containing the unit. A linear map $E: \mathcal{A} \rightarrow \mathcal{B}$ is a conditional expectation if

$$
E[b]=b \quad \forall b \in \mathcal{B}
$$

and

$$
E\left[b_{1} a b_{2}\right]=b_{1} E[a] b_{2} \quad \forall a \in \mathcal{A}, \quad \forall b_{1}, b_{2} \in \mathcal{B} .
$$

An operator-valued probability space consists of $\mathcal{B} \subset \mathcal{A}$ and a conditional expectation $E: \mathcal{A} \rightarrow \mathcal{B}$. Then, random variables $x_{i} \in \mathcal{A}(i \in I)$ are free with respect to $E$ (or free with amalgamation over $\mathcal{B}$ ) if $E\left[a_{1} \cdots a_{n}\right]=0$ whenever $a_{i} \in \mathcal{B}\left\langle x_{j(i)}\right\rangle$ are polynomials in some $x_{j(i)}$ with coefficients from $\mathcal{B}$ and $E\left[a_{i}\right]=0$ for all $i$ and $j(1) \neq j(2) \neq \cdots \neq j(n)$.

For a random variable $x \in \mathcal{A}$, we define the operator-valued Cauchy transform:

$$
G(b):=E\left[(b-x)^{-1}\right] \quad(b \in \mathcal{B}),
$$

whenever $(b-x)$ is invertible in $\mathcal{B}$.

In order to have some nice analytic behaviour, we will in the following assume that both $\mathcal{A}$ and $\mathcal{B}$ are $C^{*}$-algebras; $\mathcal{B}$ will usually be of the form $\mathcal{B}=M_{N}(\mathbb{C})$, the $N \times N$-matrices. In such a setting and for $x=x^{*}$, this $G$ is well-defined and a nice analytic map on the operator-valued upper halfplane:

$$
\mathbb{H}^{+}(B):=\left\{b \in B \mid\left(b-b^{*}\right) /(2 i)>0\right\}
$$

and it allows to give a nice description for the sum of two free selfadjoint elements. In the following we will use the notation

$$
h(b):=\frac{1}{G(b)}-b .
$$

Theorem 1 ([2]). Let $x$ and $y$ be selfadjoint operator-valued random variables free over $\mathcal{B}$. Then there exists a Fréchet analytic map $\omega: \mathbb{H}^{+}(\mathcal{B}) \rightarrow \mathbb{H}^{+}(\mathcal{B})$ so that

$$
G_{x+y}(b)=G_{x}(\omega(b)) \text { for all } b \in \mathbb{H}^{+}(\mathcal{B}) .
$$

Moreover, if $b \in \mathbb{H}^{+}(\mathcal{B})$, then $\omega(b)$ is the unique fixed point of the map

$$
f_{b}: \mathbb{H}^{+}(\mathcal{B}) \rightarrow \mathbb{H}^{+}(\mathcal{B}), \quad f_{b}(w)=h_{y}\left(h_{x}(w)+b\right)+b,
$$

and

$$
\omega(b)=\lim _{n \rightarrow \infty} f_{b}^{\circ n}(w) \quad \text { for any } w \in \mathbb{H}^{+}(\mathcal{B})
$$




\section{The Linearization Philosophy}

As we just have seen, we can deal with the sum of free selfadjoint elements, even on the operator-valued level. However, what we are interested in are polynomials in free variables, on a scalar-valued level. The relation between these two problems is given by the linearization philosophy: in order to understand polynomials $p$ in non-commuting variables, it suffices to understand matrices $\hat{p}$ of linear polynomials in those variables.

In the context of free probability this idea can be traced back to the early papers of Voiculescu; it became very prominent and concrete in the seminal work [8] of Haagerup and Thorbjørnsen on the largest eigenvalue of polynomials in independent Gaussian random matrices. A more streamlined version of this, based on the notion of Schur complement, which also has the important additional feature that it preserves selfadjointness, is due to Anderson [1]

Actually, those ideas were also used before in the context of automata theory and formal languages or non-commutative rational functions, where they go under different names, like descriptor realization. Some relevant literature in this context is 15, 3, 10. Actually, from this context it becomes clear that we cannot only linearize polynomials, but also non-commutative rational functions.

The crucial point is that for any selfadjoint polynomial $p$ there exists a linearization $\hat{p}$, which is also selfadjoint. Since $\hat{p}$ is linear it is the sum of operator-valued variables.

We will present the details of this procedure with the help of a concrete example. Let us consider the polynomial $p(x, y)=x y+y x+x^{2}$ in the free variables $x$ and $y$.

This $p$ has a linearization

$$
\hat{p}=\left(\begin{array}{crc}
0 & x & y+\frac{x}{2} \\
x & 0 & -1 \\
y+\frac{x}{2} & -1 & 0
\end{array}\right),
$$

which means that the Cauchy transform of $p$ can be recovered from the operatorvalued Cauchy transform of $\hat{p}$, namely we have

$$
G_{\hat{p}}(b)=\mathrm{id} \otimes \varphi\left((b-\hat{p})^{-1}\right)=\left(\begin{array}{cc}
\varphi\left((z-p)^{-1}\right) & \cdots \\
\cdots & \cdots
\end{array}\right) \quad \text { for } \quad b=\left(\begin{array}{ccc}
z & 0 & 0 \\
0 & 0 & 0 \\
0 & 0 & 0
\end{array}\right) .
$$

But this $\hat{p}$ can now be written as

$$
\hat{p}=\left(\begin{array}{ccc}
0 & x & \frac{x}{2} \\
x & 0 & -1 \\
\frac{x}{2} & -1 & 0
\end{array}\right)+\left(\begin{array}{ccc}
0 & 0 & y \\
0 & 0 & 0 \\
y & 0 & 0
\end{array}\right)=\hat{X}+\hat{Y}
$$

and hence is the sum of two selfadjoint variables $\hat{X}$ and $\hat{Y}$, which are, by basic properties of freeness, free over $M_{3}(\mathbb{C})$. So we can use our subordination result in order to calculate the Cauchy transform of $p$.

$$
\left(\begin{array}{cc}
\varphi\left((z-p)^{-1}\right) & \cdots \\
\cdots & \cdots
\end{array}\right)=G_{\hat{p}}(b)=G_{\hat{X}+\hat{Y}}(b)=G_{\hat{X}}(\omega(b)),
$$

where we calculate $\omega(b)$ via iterations as in Theorem 1. Figure 3 shows the agreement between the achieved theoretic result and the histogram of eigenvalues from Fig. 2 , 

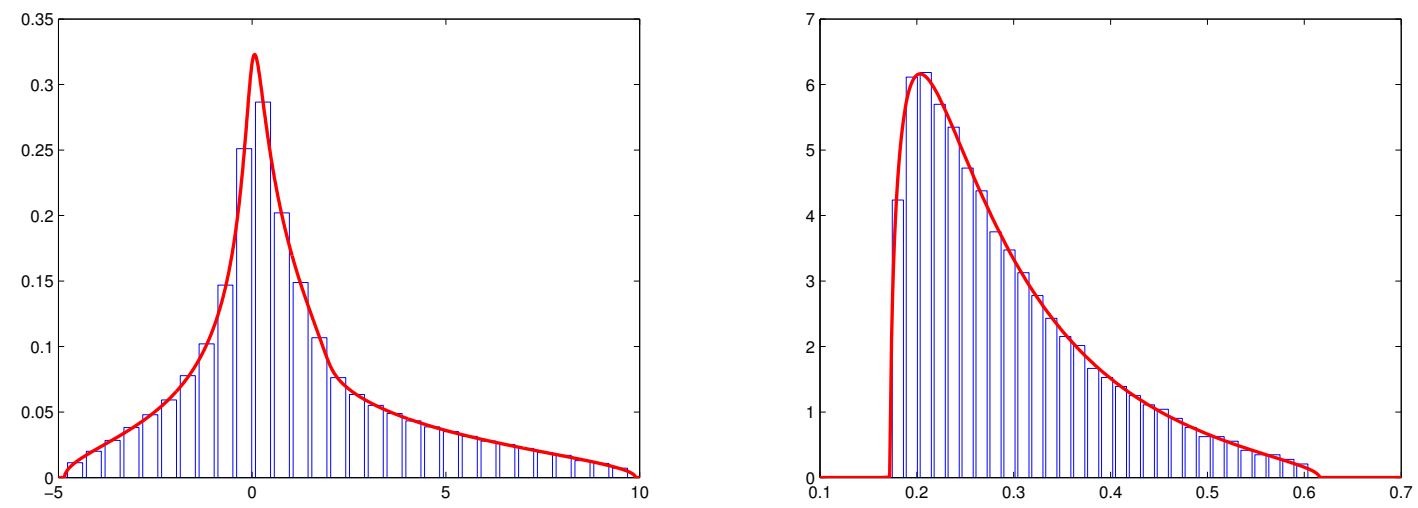

Figure 3. Plots of the distribution of $p(x, y)=x y+y x+x^{2}$ (left) for free $x, y$, where $x$ is semicircular and $y$ Marchenko-Pastur, and of the rational function $r\left(x_{1}, x_{2}\right)$ (right) for free semicircular elements $x_{1}$ and $x_{2}$; in both cases our theoretical limit curve is compared with the histogram of the corresponding eigenvalue problem; for the left case compare Figure 2

Conclusion: the combination of linearization and operator-valued subordination allows to deal with case of selfadjoint polynomials in free variables, and thus with selfadjoint polynomials in asymptotically free random matrices.

\section{The Case of Rational Functions}

As we mentioned before this linearization procedure works as well in the case of non-commutative rational functions (and this was actually the main object of interest in the work of Schützenberger). This is work in progress with Mai, let us here just give an example for this.

Consider the following selfadjoint rational function

$$
r\left(x_{1}, x_{2}\right)=\left(4-x_{1}\right)^{-1}+\left(4-x_{1}\right)^{-1} x_{2}\left(\left(4-x_{1}\right)-x_{2}\left(4-x_{1}\right)^{-1} x_{2}\right)^{-1} x_{2}\left(4-x_{1}\right)^{-1}
$$

in two free variables $x_{1}$ and $x_{2}$. The fact that we can write it as

$$
r\left(x_{1}, x_{2}\right):=\left(\begin{array}{ll}
\frac{1}{2} & 0
\end{array}\right)\left(\begin{array}{cc}
1-\frac{1}{4} x_{1} & -\frac{1}{4} x_{2} \\
-\frac{1}{4} x_{2} & 1-\frac{1}{4} x_{1}
\end{array}\right)^{-1}\left(\begin{array}{c}
\frac{1}{2} \\
0
\end{array}\right)
$$

gives us immediately a selfadjoint linearization of the form

$$
\begin{aligned}
\hat{r}\left(x_{1}, x_{2}\right) & =\left(\begin{array}{ccc}
0 & \frac{1}{2} & 0 \\
\frac{1}{2} & -1+\frac{1}{4} x_{1} & \frac{1}{4} x_{2} \\
0 & \frac{1}{4} x_{2} & -1+\frac{1}{4} x_{1}
\end{array}\right) \\
& =\left(\begin{array}{ccc}
0 & \frac{1}{2} & 0 \\
\frac{1}{2} & -1+\frac{1}{4} x_{1} & 0 \\
0 & 0 & -1+\frac{1}{4} x_{1}
\end{array}\right)+\left(\begin{array}{ccc}
0 & 0 & 0 \\
\frac{1}{2} & 0 & \frac{1}{4} x_{2} \\
0 & \frac{1}{4} x_{2} & 0
\end{array}\right) .
\end{aligned}
$$

In Figure 3 , we compare the histogram of eigenvalues of $r\left(X_{1}, X_{2}\right)$ for one realization of independent Gaussian random matrices $X_{1}, X_{2}$ of size $1000 \times 1000$ with the 
distribution of $r\left(x_{1}, x_{2}\right)$ for free semicircular elements $x_{1}, x_{2}$, calculated according to our algorithm.

\section{The Brown Measure for Non-Normal Operators}

The main point of this note is that we can actually also extend the previous method to deal with not necessarily self-adjoint polynomials in free variables.

The first problem in this situation is that we need to describe a measure on the complex plane by some analytic object. Let us first have a look on this.

For a measure $\mu$ on $\mathbb{C}$ its Cauchy transform

$$
G_{\mu}(\lambda)=\int_{\mathbb{C}} \frac{1}{\lambda-z} d \mu(z)
$$

is well-defined everywhere outside a set of $\mathbb{R}^{2}$-Lebesgue measure zero, however, it is analytic only outside the support of $\mu$.

The measure $\mu$ can be extracted from its Cauchy transform by the formula (understood in distributional sense)

$$
\mu=\frac{1}{\pi} \frac{\partial}{\partial \bar{\lambda}} G_{\mu}(\lambda)
$$

A better approach to this is by regularization:

$$
G_{\epsilon, \mu}(\lambda)=\int_{\mathbb{C}} \frac{\bar{\lambda}-\bar{z}}{\epsilon^{2}+|\lambda-z|^{2}} d \mu(z)
$$

is well-defined for every $\lambda \in \mathbb{C}$. By sub-harmonicity arguments

$$
\mu_{\epsilon}=\frac{1}{\pi} \frac{\partial}{\partial \bar{\lambda}} G_{\epsilon, \mu}(\lambda)
$$

is a probability measure on the complex plane and one has the weak convergence $\lim _{\epsilon \rightarrow 0} \mu_{\epsilon}=\mu$.

Our general polynomial in free variables will in general not be selfadjoint nor normal. Thus we need a generalization of the distribution of a selfadjoint operator to a non-normal situation. This can be done, in a tracial setting, by imitating essentially the ideas from above.

So let us consider a general (not necessarily normal) operator $x$ in a tracial noncommutative probability space $(\mathcal{A}, \varphi)$. (We need some nice analytic frame here, so $\mathcal{A}$ should be a von Neumann algebra.) We put

$$
G_{\epsilon, x}(\lambda):=\varphi\left((\lambda-x)^{*}\left((\lambda-x)(\lambda-x)^{*}+\epsilon^{2}\right)^{-1}\right) .
$$

Then

$$
\mu_{\epsilon, x}=\frac{1}{\pi} \frac{\partial}{\partial \bar{\lambda}} G_{\epsilon, \mu}(\lambda)
$$

is a probability measure on the complex plane, which converges weakly for $\epsilon \rightarrow 0$. This limit $\mu_{x}:=\lim _{\epsilon \rightarrow 0} \mu_{\epsilon, x}$ is called the Brown measure of $x$; it was introduced by L. Brown in 1986 and revived in 2000 by Haagerup and Larsen [7, who made decisive use of it for their investigations around the invariant subspace problem. 


\section{Hermitization Method}

The idea of the hermitization method is to treat non-normal operators (or random matrices) $x$ by studying sufficiently many selfadjoint $2 \times 2$ matrices built out of $x$. A contact of this idea with the world of free probabilty was made on a formal level in the works of Janik, Nowak, Papp, Zahed [11] and of Feinberg, Zee [6]. We show in 4 that operator-valued free probability is the right frame to deal with this rigorously. By combining this with our subordination formulation of operatorvalued free convolution we can then calculate the Brown measure of any polynomial in free variables.

In order to get the Brown measure of $x$, we need

$$
G_{\epsilon, x}(\lambda)=\varphi\left((\lambda-x)^{*}\left((\lambda-x)(\lambda-x)^{*}+\epsilon^{2}\right)^{-1}\right) .
$$

Let

$$
X=\left(\begin{array}{cc}
0 & x \\
x^{*} & 0
\end{array}\right) \in M_{2}(\mathcal{A})
$$

Note that $X$ is selfadjoint. Consider $X$ in the $M_{2}(\mathbb{C})$-valued probability space with repect to $E=$ id $\otimes \varphi: M_{2}(\mathcal{A}) \rightarrow M_{2}(\mathbb{C})$ given by

$$
E\left[\left(\begin{array}{ll}
a_{11} & a_{12} \\
a_{21} & a_{22}
\end{array}\right)\right]=\left(\begin{array}{ll}
\varphi\left(a_{11}\right) & \varphi\left(a_{12}\right) \\
\varphi\left(a_{21}\right) & \varphi\left(a_{22}\right)
\end{array}\right) .
$$

For the argument

$$
\Lambda_{\epsilon}=\left(\begin{array}{cc}
i \epsilon & \lambda \\
\bar{\lambda} & i \epsilon
\end{array}\right) \in M_{2}(\mathbb{C})
$$

consider now the $M_{2}(\mathbb{C})$-valued Cauchy transform of $X$

$$
G_{X}\left(\Lambda_{\varepsilon}\right)=E\left[\left(\Lambda_{\epsilon}-X\right)^{-1}\right]=\left(\begin{array}{ll}
g_{\epsilon, \lambda, 11} & g_{\epsilon, \lambda, 12} \\
g_{\epsilon, \lambda, 21} & g_{\epsilon, \lambda, 22}
\end{array}\right) .
$$

One can easily check that $\left(\Lambda_{\epsilon}-X\right)^{-1}$ is actually given by

$$
\left(\begin{array}{cc}
-i \epsilon\left((\lambda-x)(\lambda-x)^{*}+\epsilon^{2}\right)^{-1} & (\lambda-x)\left((\lambda-x)^{*}(\lambda-x)+\epsilon^{2}\right)^{-1} \\
(\lambda-x)^{*}\left((\lambda-x)(\lambda-x)^{*}+\epsilon^{2}\right)^{-1} & -i \epsilon\left((\lambda-x)^{*}(\lambda-x)+\epsilon^{2}\right)^{-1}
\end{array}\right),
$$

and thus we are again in the situation that our quantity of interest is actually one entry of an operator-valued Cauchy transform:

$$
g_{\epsilon, \lambda, 12}=G_{\varepsilon, x}(\lambda) .
$$

\section{Calculation of the Brown Measure}

So in order to calculate the Brown measure of some polynomial $p$ we should first hermitize the problem by going over to selfadjoint $2 \times 2$ matrices over our underlying space, then we should linearize the problem on this level and use finally our subordination description of operator-valued free convolution to deal with this linear problem. It might be not so clear whether hermitization and linearisation go together well, but this is indeed the case. Essentially we do here a linearization of an operator-valued model instead of a scalar-valued one: we have to linearize a polynomial in matrices. But the linearization algorithm works in this case as well.

Let us illustrate this with an example.

Consider the polynomial $p=x y$ in the free selfadjoint variables $x=x^{*}$ and $y=y^{*}$. 
For the Brown measure of this we have to calculate the operator-valued Cauchy transform of

$$
P=\left(\begin{array}{cc}
0 & x y \\
y x & 0
\end{array}\right) .
$$

In order to linearize this we should first write it as a polynomial in matrices of $x$ and matrices of $y$. This can be achieved as follows:

$$
P=\left(\begin{array}{cc}
0 & x y \\
y x & 0
\end{array}\right)=\left(\begin{array}{ll}
x & 0 \\
0 & 1
\end{array}\right)\left(\begin{array}{ll}
0 & y \\
y & 0
\end{array}\right)\left(\begin{array}{ll}
x & 0 \\
0 & 1
\end{array}\right)=X Y X
$$

$P=X Y X$ is now a selfadjoint polynomial in the selfadjoint variables

$$
X=\left(\begin{array}{ll}
x & 0 \\
0 & 1
\end{array}\right) \quad \text { and } \quad Y=\left(\begin{array}{ll}
0 & y \\
y & 0
\end{array}\right)
$$

and has thus a selfadjoint linearization

$$
\left(\begin{array}{ccc}
0 & 0 & X \\
0 & Y & -1 \\
X & -1 & 0
\end{array}\right) .
$$

Pluggin in back the $2 \times 2$ matrices for $X$ and $Y$ we get finally the selfadjoint linearization of $P$ as

$$
\left(\begin{array}{cccccc}
0 & 0 & 0 & 0 & x & 0 \\
0 & 0 & 0 & 0 & 0 & 1 \\
0 & 0 & 0 & y & -1 & 0 \\
0 & 0 & y & 0 & 0 & -1 \\
x & 0 & -1 & 0 & 0 & 0 \\
0 & 1 & 0 & -1 & 0 & 0
\end{array}\right)
$$

which can be written as the sum of two $M_{6}(\mathbb{C})$-free matrices:

$$
\left(\begin{array}{cccccc}
0 & 0 & 0 & 0 & x & 0 \\
0 & 0 & 0 & 0 & 0 & 1 \\
0 & 0 & 0 & 0 & -1 & 0 \\
0 & 0 & 0 & 0 & 0 & -1 \\
x & 0 & -1 & 0 & 0 & 0 \\
0 & 1 & 0 & -1 & 0 & 0
\end{array}\right)+\left(\begin{array}{cccccc}
0 & 0 & 0 & 0 & 0 & 0 \\
0 & 0 & 0 & 0 & 0 & 0 \\
0 & 0 & 0 & y & 0 & 0 \\
0 & 0 & y & 0 & 0 & 0 \\
0 & 0 & 0 & 0 & 0 & 0 \\
0 & 0 & 0 & 0 & 0 & 0
\end{array}\right) .
$$

For calculating the Cauchy transform of this sum we can then use again our subordination algorithm for the operator-valued free convolution from Theorem 1. Putting all the steps together gives an algorithm for calculating the Brown measure of $p$.

Of course, we expect that in nice cases the eigenvalue distribution of our polynomial evaluated in independent Wigner or Wishart matrices should converge to the Brown measure of the polynomial in the corresponding free variables. However, in contrast to the selfadjoint case this is not automatic from the convergence of all relevant moments and one has to control probabilities of small eigenvalues during all the calculations. Such control have been achieved in special cases (in particular, the circular law and the single ring theorem), but in general it has to remain open for the moment.

In the following figures we give for some polynomials the Brown measure according to our algorithm and compare this with histograms of the complex eigenvalues of the corresponding polynomials in independent random matrices. 
As before, this algorithm can also be extended to rational functions in our variables. An example for the outcome of our algorithm in the case of the non-selfadjoint rational function given by

$$
q\left(x_{1}, x_{2}\right):=\left(\begin{array}{ll}
0 & \frac{1}{2}
\end{array}\right)\left(\begin{array}{cc}
1-\frac{1}{4} x_{1} & -i x_{2} \\
-\frac{1}{4} x_{2} & 1-\frac{1}{4} x_{1}
\end{array}\right)^{-1}\left(\begin{array}{l}
\frac{1}{2} \\
0
\end{array}\right)
$$

is shown in Figure 7
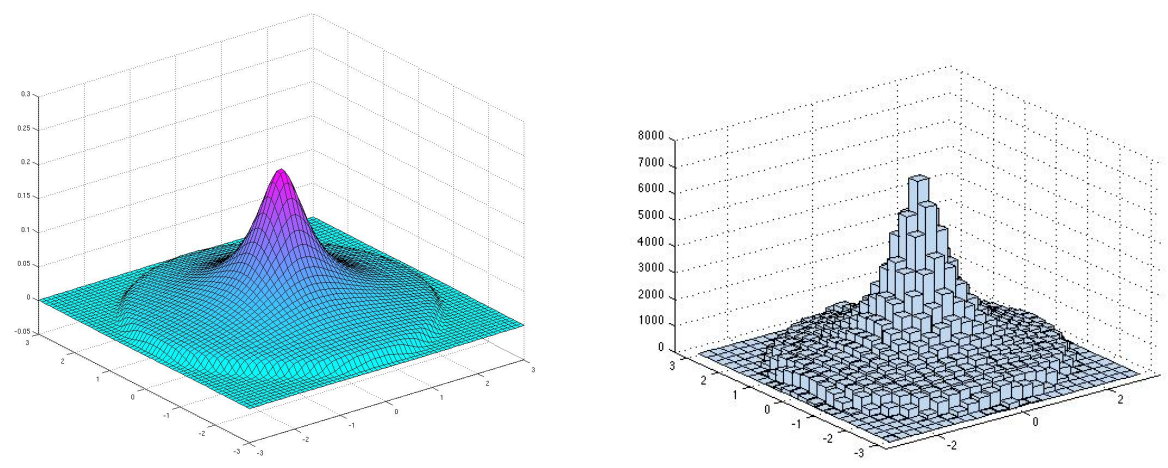

Figure 4. Brown measure (left) of $p(x, y, z)=x y z-2 y z x+z x y$ with $x, y, z$ free semicircles, compared to histogram (right) of the complex eigenvalues of $p(X, Y, Z)$ for independent Wigner matrices with $N=1000$
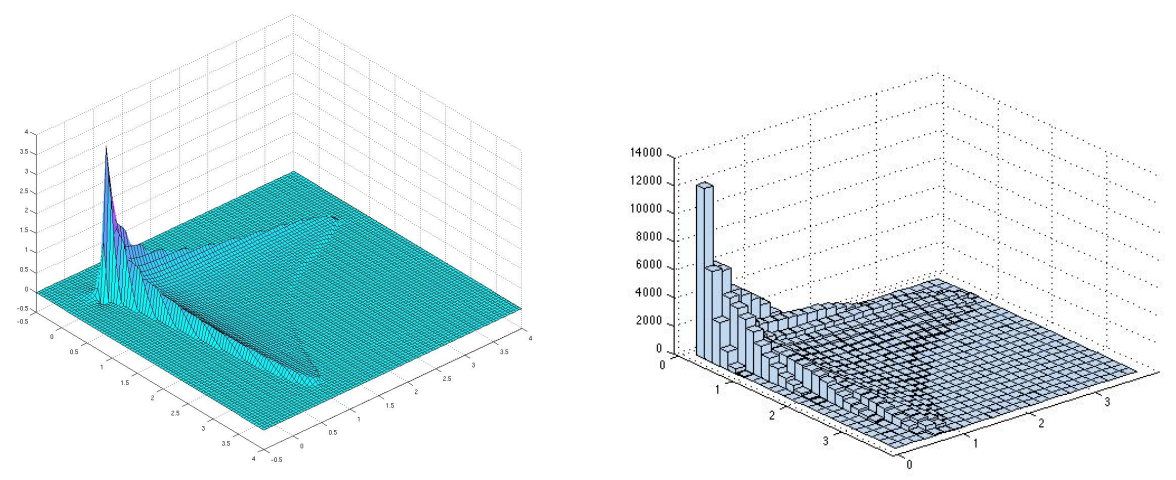

FiguRE 5. Brown measure (left) of $p(x, y)=x+i y$ with $x, y$ free semicircles, compared to histogram (right) of the complex eigenvalues of $p(X, Y)$ for independent Wigner matrices $X$ and $Y$ with $N=1000$ 

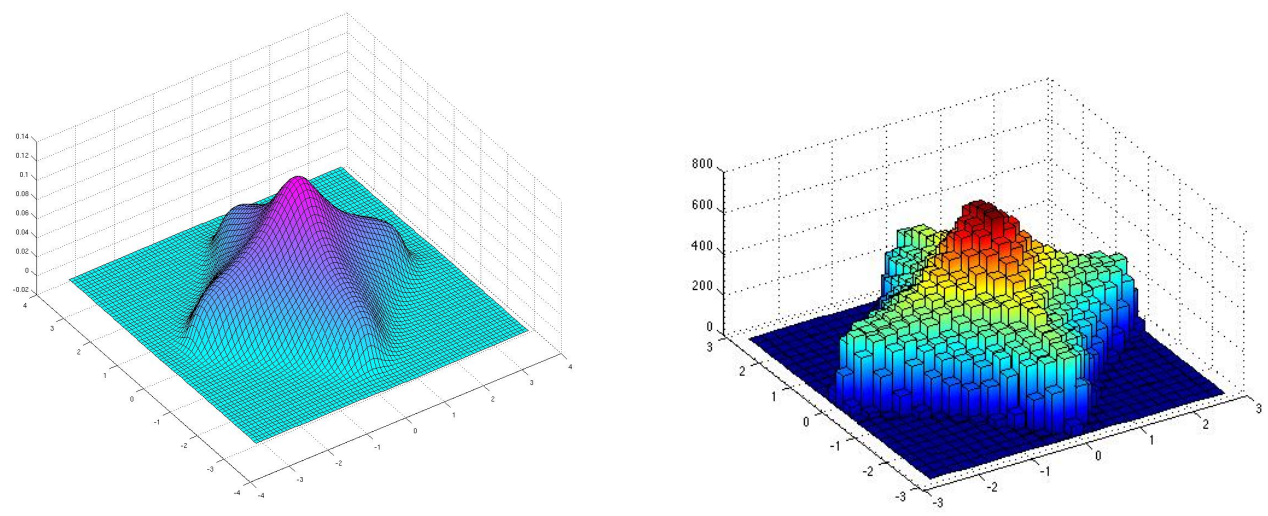

Figure 6. Brown measure (left) of $p\left(x_{1}, x_{2}, x_{3}, x_{4}\right)=x_{1} x_{2}+$ $x_{2} x_{3}+x_{3} x_{4}+x_{4} x_{1}$ with $x_{1}, x_{2}, x_{3}, x_{4}$ free semicircles, compared to histogram (right) of the complex eigenvalues of $p\left(X_{1}, X_{2}, X_{3}, X_{4}\right)$ for independent Wigner matrices $X_{1}, X_{2}, X_{3}, X_{4}$ with $N=1000$

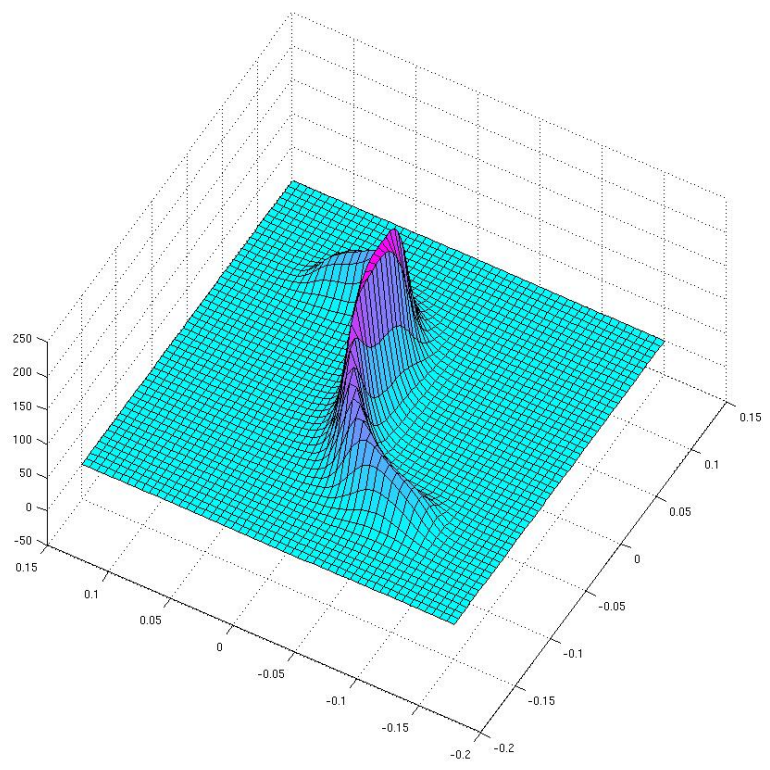

Figure 7. Brown measure of the non-selfadjoint polynomial $q\left(x_{1}, x_{2}\right)$ for free semicircular elements $x_{1}$ and $x_{2}$

\section{REFERENCES}

[1] G. W. Anderson, Convergence of the largest singular value of a polynomial in independent Wigner matrices, Ann. Probab. 41 (2013), no. 3B, 2103-2181. 
[2] S.T. Belinschi, T. Mai, and R. Speicher, Analytic subordination theory of operator-valued free additive convolution and the solution of a general random matrix problem, Preprint, 2013, arXiv:1303.3196v3, to appear in CRELLE J. Reine Angew. Math.

[3] J. Berstel and C. Reutenauer, Rational Series and their Languages, EATCS Monographs on Theoretical Computer Science, Springer, 1984.

[4] S. T. Belinschi, P. Sniady, and R. Speicher, Asymptotic eigenvalue distribution of nonhermitian random matrices and Brown measure of non-normal operators: hermitian reduction and linearization method, in preparation.

[5] R. Couillet and M. Debbah, Random matrix methods for wireless communications, Cambridge University Press, 2011.

[6] J. Feinberg and A. Zee, Non-Hermitian random matrix theory: method of Hermitian reduction, Nuclear Phys. B 504 (1997), 579-608.

[7] U. Haagerup and F. Larsen Brown's spectral distribution measure for R-diagonal elements in finite von Neumann algebras, J. Funct. Anal. 176 (2000), 331-367.

[8] U. Haagerup and S. Thorbjørnsen, A new application of Random Matrices: $\operatorname{Ext}\left(C_{\mathrm{red}}^{*}\left(F_{2}\right)\right)$ is not a group, Annals of Mathematics 162 (2005), 711-775.

[9] W. Hechem, P. Loubaton, and J. Najim, Deterministic equivalents for certain functionals of large random matrices, Ann. Appl. Probab. 17 (2007), 875-930.

[10] J. W. Helton, S. A. McCullough, and V. Vinnikov, Noncommutative convexity arises from linear matrix inequalities, J. Funct. Anal. 240 (2006), no. 1, 105-191.

[11] R. Janik, M. Nowak, G. Papp, and I. Zahed, Non-Hermitian random matrix models, Nuclear Phys. B 501 (1997), 603-642.

[12] V. Marchenko and L. Pastur, Distributions of eigenvalues for some sets of random matrices, Math. USSR-Sbornik 1 (1967), 457-483.

[13] A. Nica and R. Speicher, Commutators of free random variables. Duke Math. J. 92 (1998), no. $3,553-592$.

[14] A. Nica and R. Speicher, Lectures on the combinatorics of free probability. Cambridge University Press, 2006.

[15] M. P. Schützenberger, On the Definition of a Family of Automata. Information and Control 4 (1961), 245-270.

[16] R. Speicher and C. Vargas, Free deterministic equivalents, rectangular random matrix models, and operator-valued free probability theory, Random Matrices: Theory and Applications 1 (2012), 1150008 (26 pages)

[17] A. Tulino and S. Verdù, Random Matrix Theory and Wireless Communications, Foundations and Trends in Communication and Information Theory, 1, 2004.

[18] V. Vasilchuk, On the asymptotic distribution of the commutator and anticommutator of random matrices, J. Math. Phys. 44 (2003), no. 4, 1882-1908.

[19] D. Voiculescu, Addition of certain non-commutative random variables. J. Funct. Anal. 66 (1986), 323-346.

[20] D. Voiculescu, Limit laws for random matrices and free products. Invent. Math. 104 (1991), 201-220.

[21] D. Voiculescu, Operations on certain non-commutative operator-valued random variables, Astérisque (1995), no. 232, 243-275, Recent advances in operator algebras (Orléans, 1992).

FACHRICHTUNG MATHEMATIK, SAARLAND UNIVERSITY

E-mail address: SPEICHER@MATH.UNI-SB.DE 